\title{
LA PLANIFICACIÓN DE LAS ACTIVIDADES DE LOS ESTUDIANTES EN LOS TRABAJOS PRÁCTICOS DE FÍSICA: ANÁLISIS DE PRÁCTICAS DE PROFESORES
}

\author{
RICHOUX, HÉLÈNE ${ }^{1}$ y BEAUFILS, DANIEL ${ }^{2}$ \\ ${ }^{1}$ Département de Didactique des Disciplines. Institut National de Recherche Pédagogique. 29 rue d'Ulm \\ F-75230 Paris cedex 05 \\ marie-hele.richoux@ac-versailles.fr \\ ${ }^{2}$ DidaScO. Université Paris XI. Bâtiment 333. F-91405 Orsay cedex \\ daniel.beaufils@df.cso.u-psud.fr
}

\begin{abstract}
Resumen. Los trabajos prácticos ocupan un lugar importante en la enseñanza de la física y de la química y son generalmente presentados como el lugar de realización de actividades científicas. Este artículo es un estudio de la elaboración de trabajos prácticos por parte de profesores de secundaria, realizado sobre la base de entrevistas y de observaciones de clases. Un primer análisis muestra que los profesores no hacen en realidad ninguna referencia a la actividad científica y que la planificación de la sesión recibe la máxima atención. La elaboración de una parrilla específica ha permitido un análisis detallado que muestra una coherencia fuerte entre planificación y «razones» invocadas por los profesores. Proponemos como síntesis un modelo de funcionamiento del «profesor-constructor».

Palabras clave. Profesorado, física, trabajo de laboratorio, nivel secundario.
\end{abstract}

Summary. Practical work is usually considered as the way to do science and to learn about experimental inquiry. The work we report in this paper focuses on the way physics teachers are designing practical work at secondary level. Analises of interviews to teachers and observations of labwork sessions show that teachers never invoke scientific processes, but give chief importance to planning considerations. We designed a specific grid for a more detailed analysis. Results show a great coherence between planning and the reasons given by teachers. We propose, as a synthesis, a model for the performance of the «designer-teacher».

Keywords. Teachers, physics, labwork, secondary level.

En Francia, desde 1902, las sesiones de trabajos prácticos en las cuales los estudiantes manipulan ellos mismos el material (Belhoste et al., 1996) ocupan una parte importante del tiempo dedicado a la enseñanza de la física y la química y son consideradas esenciales para la adquisición de conocimientos conceptuales y procedimentales. Así, los objetivos de los programas actuales recuerdan que «se concede un lugar privilegiado a las actividades experimentales [...]. Estas actividades permiten, en efecto, determinar la relación particular que las ciencias experimentales establecen con el mundo real, de la cual se desprenden una visión y una comprensión unificadas de fenómenos a priori muy diversos» (Boletín Oficial de la Educación Nacional, 1999). Desde hace años, hay numerosos trabajos acerca de la naturaleza y el papel de los trabajos prácticos, tanto en Francia (Guillon, 1995; Bécu-Robinault, 1997; Beney y Séré, 2001) como en el ámbito internacional (Séré, 1998; Leach y Paulsen, 1999). En este artículo hemos enfocado nuestra atención sobre el diseño de trabajos prácticos por parte de los profesores, investigando los elementos que ellos tienen en cuenta y las razones de sus elecciones. Proponemos como síntesis un modelo de funcionamiento del «profesorconstructor». 


\section{MARCO TEÓRICO}

\section{Problemática}

La estructura «clásica» de los trabajos prácticos se apoya sobre el hecho de poner a disposición de los estudiantes una ficha de actividades y aparatos adecuados para estudiar diferentes fenómenos, generalmente de forma cuantitativa (mediciones, tratamientos numéricos, modelización). En un gran número de casos, este procedimiento se apoya sobre la hipótesis de una cierta «autonomía» de los estudiantes: durante una hora y media, el estudiante es confrontado con la realidad de la experiencia, debiendo a su vez poner en acción los conocimientos adquiridos en el curso y así consolidarlos, o bien adquirir otros (saber y saber hacer del físico o del químico).

Cuando el profesor diseña la sesión de trabajos prácticos, plantea para sus estudiantes objetivos particulares de aprendizaje y de estructuración del conocimiento; tiene la hipótesis implícita de que las actividades que ellos hacen producirán los efectos esperados para la adquisición de conocimientos. El profesor construye un medio de interacción específico para los estudiantes, con un objetivo preciso.

\section{Medio didáctico y teoría de situaciones}

Nuestro enfoque se fundamenta en la didáctica de las matemáticas y, más específicamente, en el concepto de medio, definido por Brousseau (1988, p. 320) en el marco de la «teoría de situaciones» (Brousseau, 1986) como el sistema con el cual el estudiante interactúa. Siguiendo a este autor, debemos considerar la existencia de unas situaciones donde los aprendizajes tienen lugar sin que la intención didáctica aparezca en las actividades (estas situaciones son llamadas adidácticas), y de otras, por el contrario, donde las actividades están fundamentadas en métodos y objetivos didácticos explícitos (estas situaciones y medios son calificados de didácticos). Esta diferenciación cobra pleno sentido en la enseñanza de la física y la química, ya que, por un lado, hay actividades propias de laboratorios científicos que son transpuestas al aula (Guillon, 1995) y en las que los estudiantes «harían ciencia» (situaciones adidácticas: Tsoumpélis y Gréa, 1995), mientras que, por otra parte, por lo que hemos visto en nuestro estudio, si bien en las sesiones de trabajos prácticos «tradicionales» los estudiantes manipulan dispositivos científicos y efectúan modelizaciones, las actividades con mediciones no tienen generalmente ni el rigor científico ni toda la extensión posible en relación con las actividades de los físicos: las actividades llamadas científicas corresponden entonces a situaciones didácticas.

Para precisar lo que consideramos como «medio» del estudiante en las sesiones de trabajos prácticos, hacemos referencia a Brousseau, citado por Perrin-Glorian (1994, p. 129): «[...] en una situación de acción, llamamos medio a todo aquello que actúa sobre el estudiante o sobre lo cual el estudiante actúa. El medio puede no involucrar a un profesor o a otros estudiantes». En nuestro caso, el ambiente material mencionado antes constituye evidentemente lo esencial. Pero conviene incluir en la construcción del me- dio la «ficha de trabajos prácticos», en la cual se formulan las preguntas que deben ser contestadas por los estudiantes, los problemas que deben ser resueltos y, a menudo, indicaciones sobre los medios a utilizar. Excluir del medio a los otros estudiantes puede ser debatible, pero lo hemos aceptado en nuestra investigación porque es fácil constatar (lo veremos más tarde) que el profesor, en el diseño de la sesión, generalmente no organiza interacciones entre los estudiantes. Y por el contrario, hemos decidido incluir al profesor en el medio, puesto que, como veremos durante el análisis, él prevé sus interacciones con los estudiantes.

Además, en la educación secundaria francesa, las actividades de trabajos prácticos se inscriben en un marco muy acotado: una sesión por semana, de una hora y media de duración, invariablemente intercalada entre dos cursos, y dedicada alternativamente a la física y a la química. En consecuencia, estaba claro para nosotros que las decisiones de los profesores de proponer tal o cual experiencia, tal o cual medición o análisis, no podían derivarse únicamente de parámetros «didácticos»: para que funcione el «proceso pedagógico», el profesor debe además asegurar la factibilidad de las tareas. El lugar del trabajo de planificación de las actividades es entonces un punto importante de la estructuración del medio que se analiza.

\section{EL PROBLEMA DE LA INVESTIGACIÓN}

El deslizamiento de situaciones desde «actividades científicas» adidácticas a situaciones fuertemente didácticas que hemos mencionado más arriba está sin duda relacionado con el diseño de situaciones muy planificadas. Nuestro trabajo se ha centrado en el análisis de los conocimientos de la situación puestos en juego por los profesores (Comiti et al., 1995) y en la investigación de la coherencia en la construcción y planificación del medio de interacción. En términos de «medio» y de «situación», nuestra investigación podría concretarse en dos preguntas:

- ¿Cuáles son las razones que prevalecen en la elección de las actividades experimentales?:

- ¿razones didácticas ligadas a la física y a su aprendizaje?; o

- ¿razones pedagógicas ligadas a la organización de la enseñanza?

- ¿Cuál es el papel (naturaleza e importancia) de las restricciones de planificación en la elaboración y estructuración del medio?

\section{ELEMENTOS DE METODOLOGÍA}

De forma semejante a otros trabajos (Hewson y Olsen, 1994; Nott y Smith, 1995; Olsen et al., 1996; Luc y Durey, 1997; Haslam y Gunstone, 1998), a fin de conseguir un nivel suficientemente fino en la descripción y el análisis de las sesiones de trabajos prácticos, nos hemos centrado 
en estudios de casos. Nos situamos en el marco del análisis de prácticas de profesores de física.

\section{Elección del nivel de enseñanza y de las sesiones de trabajos prácticos. Elección de los profesores}

Hemos elegido trabajar con diferentes profesores sobre los mismos temas de trabajos prácticos de un mismo nivel de enseñanza. Nuestro estudio se centró en el análisis de situaciones «clásicas» que incluyen a priori actividades cuantitativas. Hemos optado por el nivel de la clase de première $S$ (16-17 años) a fin de eliminar la fuerte influencia de los exámenes del bachillerato sobre las decisiones de los profesores (Richoux, 1998).

Analizamos aquí el trabajo hecho con dos profesores (designados como P2 y P4) que enseñan ciencias en secundaria desde hace unos diez años. Por otra parte, hemos intentado minimizar a priori la influencia del parámetro «estudiantes» eligiendo profesores que trabajan en escuelas en las cuales el reclutamiento de los estudiantes no es ni privilegiado ni particularmente difícil.

Hemos seleccionado tres temas tratados en la mayor parte de los manuales de nivel de première $S$, reconocidos como «temas perennes» (Bécu-Robinault y Tiberghien, 1995) que, a priori, podían tener objetivos e incluir actividades diferentes:

- caída libre;

- medición de una magnitud calorimétrica; y

- estudio de un dipolo eléctrico desde el punto de vista de un balance energético.

\section{La recogida de datos}

En función de los referentes indicados en el marco teórico, hemos buscado identificar los elementos que los profesores tienen en cuenta al diseñar el medio de la sesión de trabajo experimental que se analiza. En coherencia con nuestros problemas de investigación, perseguimos obtener y estudiar la explicitación por parte de los profesores de las actividades que ellos ponen en marcha; así, antes de cada sesión los hemos entrevistado acerca de las secuencias elaboradas. Paralelamente, hemos recogido y analizado las fichas de trabajos prácticos, ya que éstas son a la vez representativas de la planificación realizada por los profesores y un elemento del medio del estudiante.

La observación de las actividades de los estudiantes y de los profesores durante la secuencia de trabajos prácticos, el registro de los intercambios verbales entre ellos y los apuntes de los alumnos nos informan sobre el funcionamiento del medio y posibilitan el contraste con la planificación elaborada por los profesores. Una entrevista realizada después de cada sesión permite a los profesores hacer su propio análisis de las eventuales diferencias entre la previsión y lo sucedido en la sesión. El análisis de las respuestas debería informarnos sobre el medio que los profe- sores han construido para los estudiantes y sobre la previsión de su funcionamiento (Ayçaguer-Richoux, 2000).

Las preguntas de la entrevista previa tratan esencialmente sobre la planificación de las actividades, sobre los comportamientos, actitudes y actividades, y sobre los resultados esperados. No hemos pedido justificaciones a los profesores: hemos intentado que nuestras preguntas no indujeran una respuesta fabricada para la circunstancia. Estas preguntas se apoyaron en las fichas redactadas por los profesores.

Todos estos datos, para cada sesión de trabajos prácticos, fueron el punto de partida para el análisis.

\section{ANÁLISIS DE LA CONSTRUCCIÓN DEL ME- DIO DEL ESTUDIANTE}

Hemos efectuado un análisis en dos tiempos. En un primer momento, hemos realizado un primer rastreo de las diferentes categorías de argumentos propuestos por los profesores. Partiendo de un cuadro amplio, que permite incluir elementos relacionados con la idea de una transposición de las actividades científicas y los elementos relacionados con la organización material y la planificación, hemos afinado las categorías e identificado las subcategorías correspondientes. En un segundo momento, hemos considerado esta categorización como una herramienta de análisis y hemos aplicado la parrilla así construida para hacer un análisis más fino y cuantitativo de las otras entrevistas.

A continuación mostramos cómo la primera fase ha permitido arrojar luz sobre aspectos esenciales de la argumentación de los profesores; luego damos resultados de conjunto para las seis sesiones estudiadas. Finalizamos este artículo proponiendo un modelo para la planificación de los trabajos prácticos que sintetice los resultados de nuestro estudio.

\section{Los elementos de análisis del discurso}

\section{Primer análisis del discurso: las diferentes categorías}

Efectuamos un primer análisis a partir de las entrevistas realizadas con los profesores $\mathrm{P} 2$ y $\mathrm{P} 4$ y de entrevistas con profesores de terminale $S$ (17-18 años). El primer tema de análisis acerca de la «planificación de las actividades» es coherente con las preguntas que les habíamos formulado en las entrevistas previas. De manera general, aunque la planificación de las actividades y las producciones de los estudiantes no genera ninguna duda en la práctica general de los profesores (Tochon, 1989) y esto se hace todavía más visible en el marco de las actividades experimentales de trabajos prácticos en las cuales frecuentemente se distribuyen fichas de actividades a los estudiantes. El segundo tema de análisis concierne a las «razones, justificaciones y argumentaciones» que dan los profesores para explicar las elecciones que ellos hacen cuando elaboran una sesión de trabajos prácticos científicos. 
Nuestra hipótesis inicial era que los «criterios de cientificidad» (es decir, elementos tales como aparatos, métodos, procedimientos, que hacen referencia a la «ciencia» o a la «actividad de los científicos») aparecen como una justificación de las elecciones realizadas. Para esta categoría, trabajamos en un primer momento sobre una estructuración alrededor de las concepciones de los profesores acerca del funcionamiento de la ciencia y acerca del aprendizaje y la enseñanza de la física (Robardet, 1998; Roletto, 1998; Van Driel y Verloop, 1999; Porlán et al., 2000). Pero claramente -y se trata para nosotros de un primer resultado esencial- las referencias esperadas a procedimientos científicos no aparecieron espontáneamente en las entrevistas; por otra parte, la articulación didáctico-científica tampoco se manifestó de manera explícita en el discurso de los profesores.

Por ello, una categorización de las razones fundamentada sobre una articulación entre los procedimientos didácticos y científicos se reveló inadecuada. Nuestra categorización comporta esencialmente tener en cuenta las referencias a la enseñanza científica y a la gestión del grupo (planificación).

\section{Los items de planificación}

Las entrevistas han hecho aparecer elementos de planificación relativos al lugar de la sesión de trabajos prácticos en relación con el programa (aspecto institucional) y en relación con la progresión seguida por los profesores en las clases particulares estudiadas. Hemos llamado a estos elementos de planificación externos, para distinguirlos de otros que pueden calificarse de internos a la sesión de trabajos prácticos y dan cuenta de la planificación de las actividades y de las producciones pero también de los problemas de organización material de la secuencia recogidos por los profesores (Fig. 1).

Al estar nuestro estudio enfocado en el papel de lo cuantitativo en los trabajos prácticos, hemos buscado analizar más finamente todo lo que concierne a las actividades de los estudiantes en este aspecto: toma de mediciones, tratamiento matemático de los datos y análisis. Esto nos ha llevado a dividir más finamente el ítem «actividades de los estudiantes» cuando construimos la parrilla de análisis (Tabla I).

\section{Los items de razón, justificación y argumentación}

Como hemos dicho antes, las referencias a las actividades científicas casi no aparecieron en el discurso de los profesores. La parrilla de análisis finalmente utilizada no indica entonces «razones científicas» como categoría principal. Por lo tanto, no distinguimos más que dos categorías, que hemos llamado razones de funcionamiento (entreprise) y razones de enseñanza.

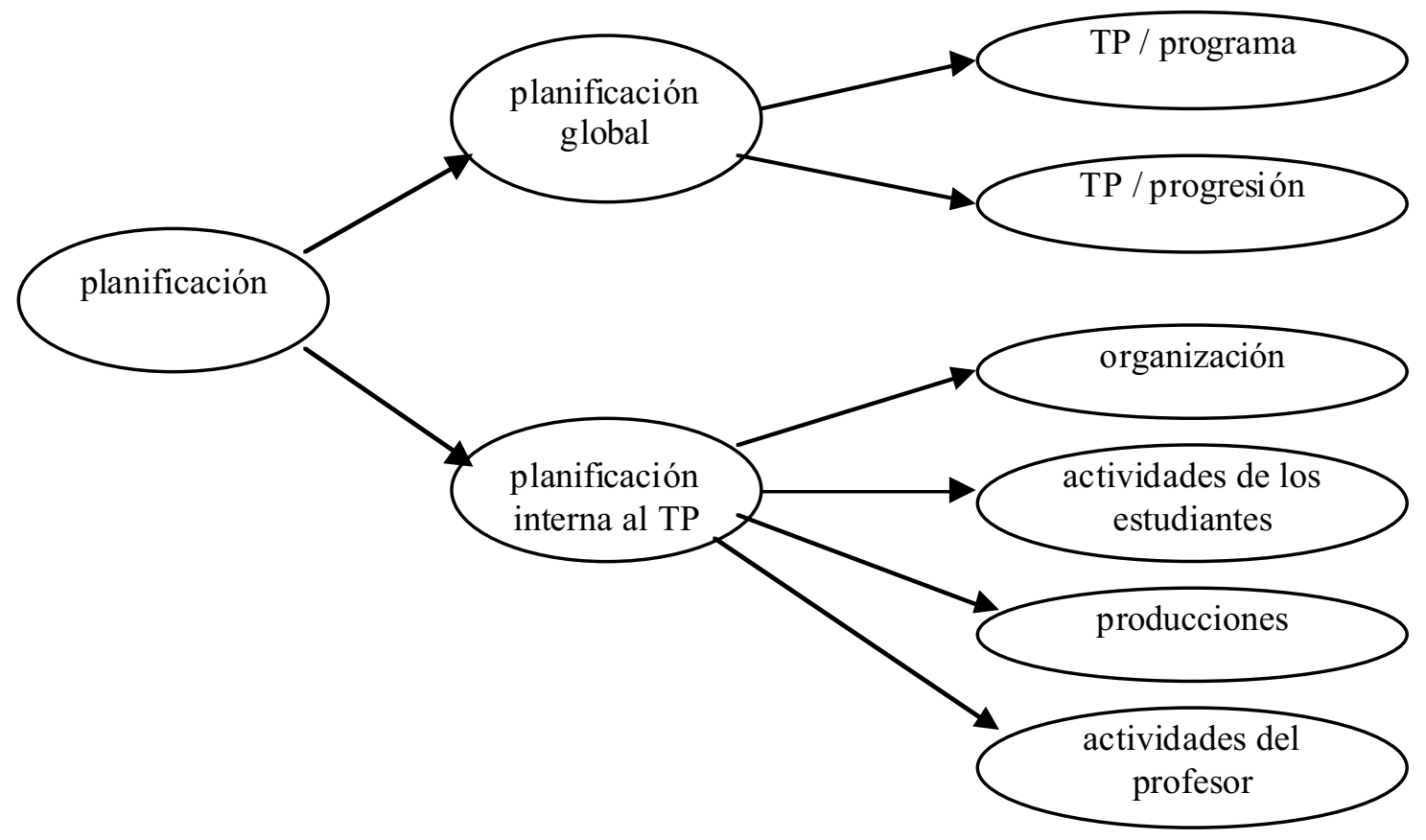




\section{Las razones de1 funcionamiento}

En esta categoría, hemos considerado las restricciones que los profesores deben tener en cuenta institucionalmente (programas, duración de las sesiones, etc.), las restricciones materiales específicas de la enseñanza experimental (material disponible, equipamiento de los laboratorios, etc.) y un punto específico de la organización de la enseñanza de las ciencias en secundaria: la necesidad de alternar las sesiones de trabajos prácticos con las clases teóricas.

\section{Las razones de enseñanza}

Esta categoría agrupa las razones correspondientes a la enseñanza y al aprendizaje, a las elecciones propias de los profesores concernientes a la sesión y a la gestión del grupo durante la actividad. Hemos distinguido entre las razones relacionadas con la «enseñanza de contenidos y procedimientos de física» y las relacionadas con «tener en cuenta el aprendizaje de la física». Por un lado, los profesores argumentan la organización de sus sesiones en función de conocimientos conceptuales y procedimentales (los clasificamos, por tanto, en la primera categoría), pero, por otro lado, pueden interesarse más específicamente en el estudiante que aprende y tener en cuenta sus dificultades (entonces hablamos de la segunda categoría).

Entre las razones relacionadas con «elecciones para la enseñanza de la física» que los profesores explicitan, destacamos la importancia que otorgan a lo que hemos llamado «construir un referente». En la categoría «objetivos», uno a destacar es el de aportar a los estudiantes un conoci- miento (observación o experiencia común) sobre el cual se podrá construir la continuidad del curso.

En la categoría «gestión de la actividad del grupo», incluimos todo los que los profesores mencionan para que los estudiantes puedan trabajar de manera «autónoma», para que ninguno quede inactivo, etc. Hemos asociado a estos argumentos aquello que son incertidumbres sobre el desarrollo de la sesión y dificultades posibles en cuanto al funcionamiento del material y a la real autonomía de los estudiantes.

En el mismo nivel hemos agregado lo que llamamos los «objetivos generales de la enseñanza», que se refieren directamente a los «objetivos no disciplinares» listados por la Inspección General de Física y Química (IGEN, 1996) para los trabajos prácticos (Fig. 2).

\section{Resultados del análisis de las entrevistas}

La siguiente parrilla de análisis (Tabla I), con las distintas categorías, la hemos utilizado para el análisis del conjunto de las entrevistas. Hemos atribuido un código a cada ítem.

Para el análisis de las transcripciones de las entrevistas previas, hemos realizado primeramente un recorte del discurso en secuencias temáticas. El cambio de tema podía ser iniciado por cualquiera de los interlocutores. También hemos realizado un segundo recorte en unidades de significado; cada unidad contiene un argumento o un elemento de planificación según la parrilla de análisis.

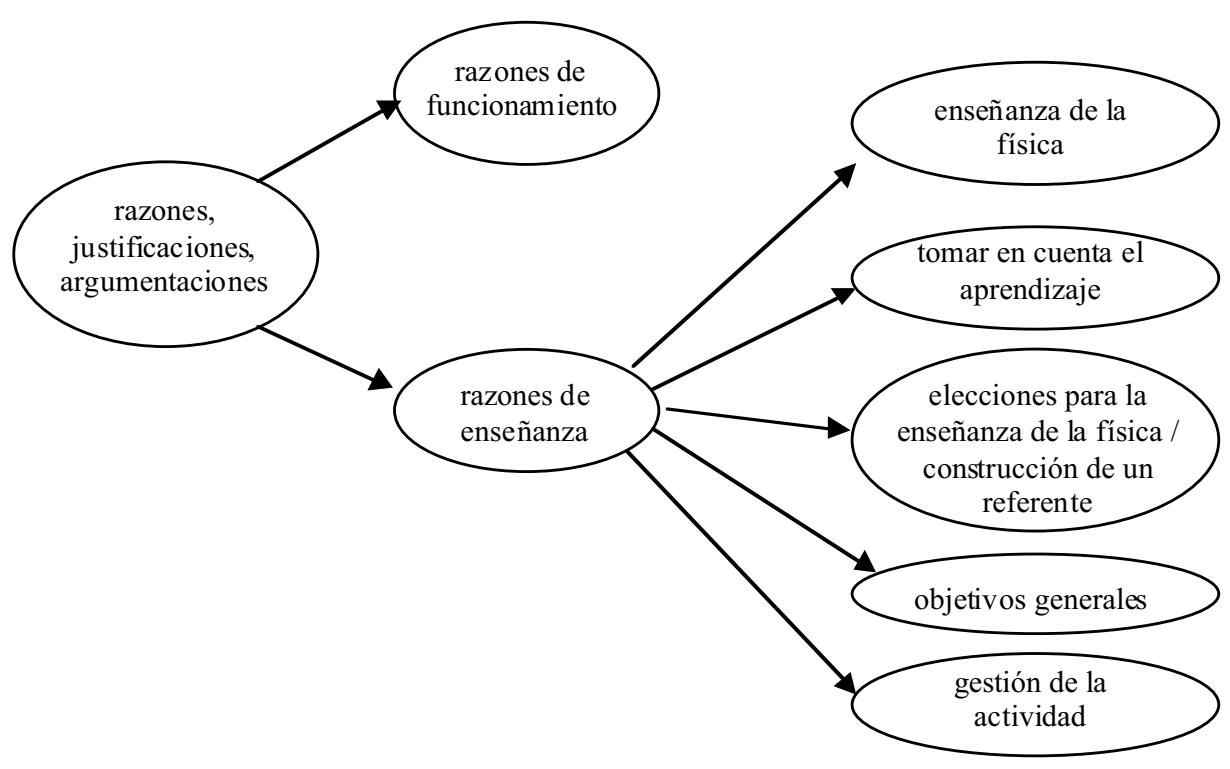


Tabla I

Parrilla de análisis completa y código de los ítems.

\begin{tabular}{|c|c|c|c|c|}
\hline & & & & Código \\
\hline \multirow{9}{*}{ Planificación } & \multirow[t]{2}{*}{ Planificación global } & Lugar del TP en relación con el programa & & $\operatorname{Pg} 1$ \\
\hline & & Lugar del TP en el curso & & $\operatorname{Pg} 2$ \\
\hline & \multirow{7}{*}{$\begin{array}{c}\text { Planificación } \\
\text { interna } \\
\text { al TP }\end{array}$} & Organización de la sesión & & Pil \\
\hline & & \multirow{4}{*}{ Actividades de los estudiantes } & $\begin{array}{c}\text { Montaje } \\
\text { y mediciones }\end{array}$ & $\mathrm{Pi} 2$ \\
\hline & & & Tratamiento & Pi3 \\
\hline & & & Análisis y discusión & Pi4 \\
\hline & & & Otros & Pi5 \\
\hline & & Producciones de los estudiantes & & Pi6 \\
\hline & & Actividad del profesor & & $\mathrm{Pi} 7$ \\
\hline
\end{tabular}

\begin{tabular}{|c|c|c|c|c|}
\hline & & Restricciones institucionales (programas, duración...) & & Re1 \\
\hline \multirow{14}{*}{ Razones } & Razones de & Medios materiales (disponibilidad, calidad...) & & $\operatorname{Re} 2$ \\
\hline & & Articulación TP/curso & & $\operatorname{Re} 3$ \\
\hline & \multirow{12}{*}{$\begin{array}{l}\text { Razones de } \\
\text { enseñanza }\end{array}$} & Enseñanza de conocimientos & & Rd1 \\
\hline & & Enseñanza de procedimientos & & $\mathrm{Rd} 2$ \\
\hline & & Enseñanza de un proceso de investigación & & $\mathrm{Rd} 3$ \\
\hline & & $\begin{array}{c}\text { Tener en cuenta las dificultades de los estudiantes } \\
\text { y el aprendizaje de la física }\end{array}$ & & $\mathrm{Rd} 4$ \\
\hline & & Construcción de un referente & & $\operatorname{Rd} 5$ \\
\hline & & Elecciones propias para la enseñanza de la física & & Rd6 \\
\hline & & \multirow[b]{4}{*}{ Gestión de la actividad } & Estudiantes activos & Rp1 \\
\hline & & & Profesor disponible & $\mathrm{Rp} 2$ \\
\hline & & & Estudiantes & Rp3 \\
\hline & & & $\begin{array}{c}\text { Incertidumbres, } \\
\text { dificultades para el } \\
\text { profesor }\end{array}$ & $\begin{array}{l}\text { Rp4a-material } \\
\text { Rp4b-estudiantes }\end{array}$ \\
\hline & & Objetivos generales de la enseñanza & & Rp5 \\
\hline & & Otras & & Rp6 \\
\hline
\end{tabular}

En una misma secuencia temática, se contabilizan las diferentes unidades de significado procurando no duplicar la codificación para las repeticiones ligadas a la argumentación. Este método nos ha permitido contar las apariciones 
Figura 3

Diagramas de frecuencias (en porcentaje) de las categorías principales.

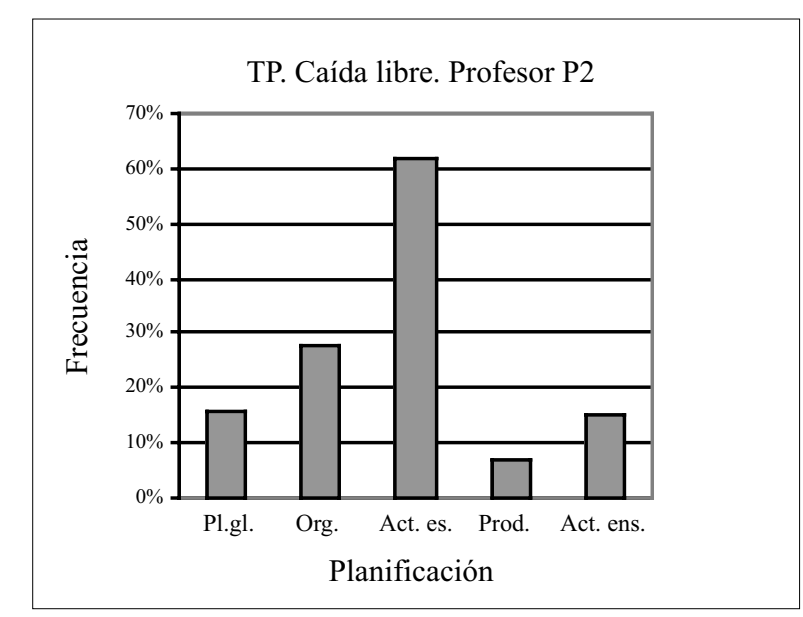

Número total de unidades codificadas: 95

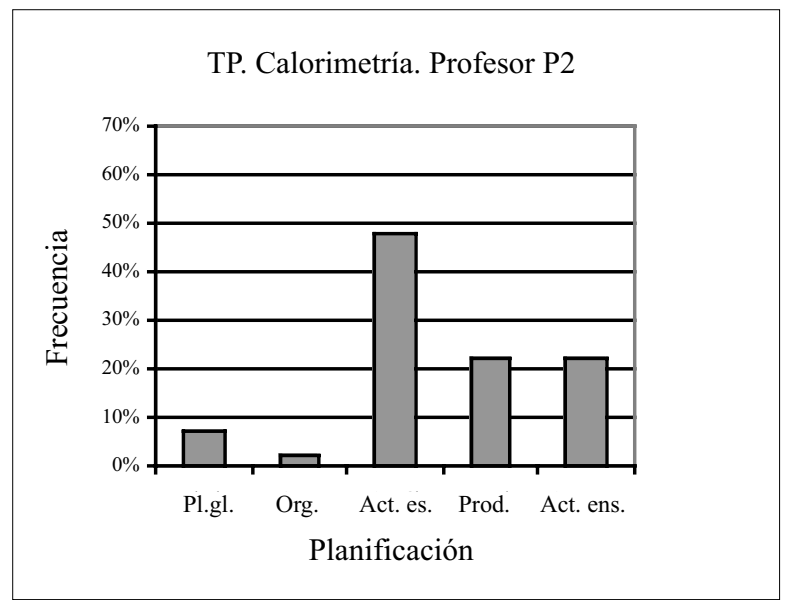

Número total de unidades codificadas: 32

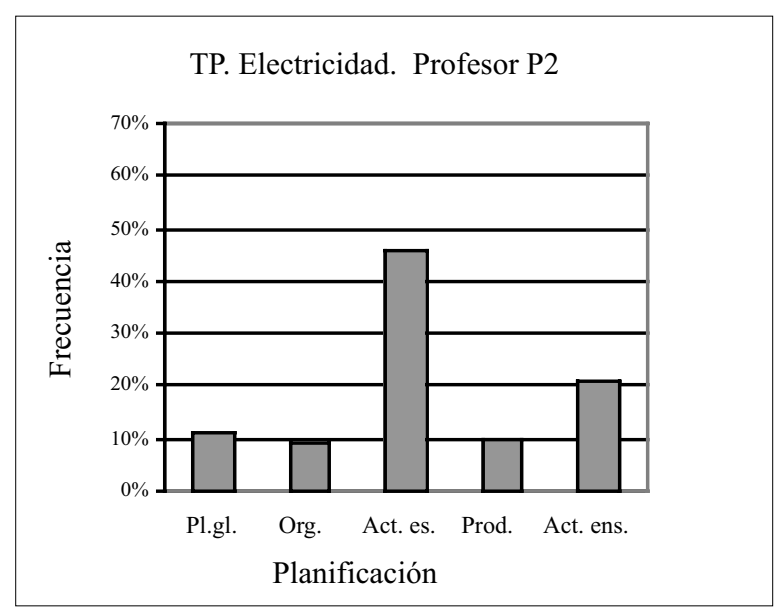

Número total de unidades codificadas: 77

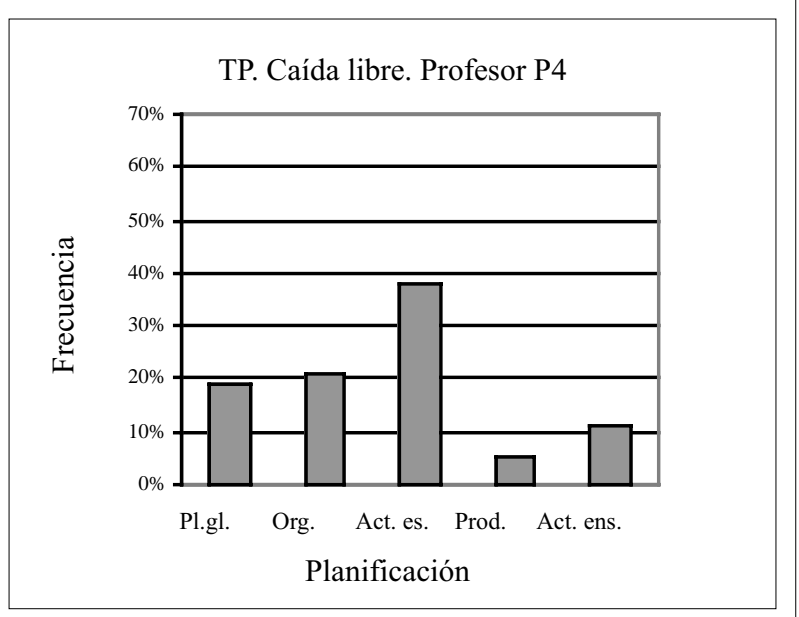

Número total de unidades codificadas: 64

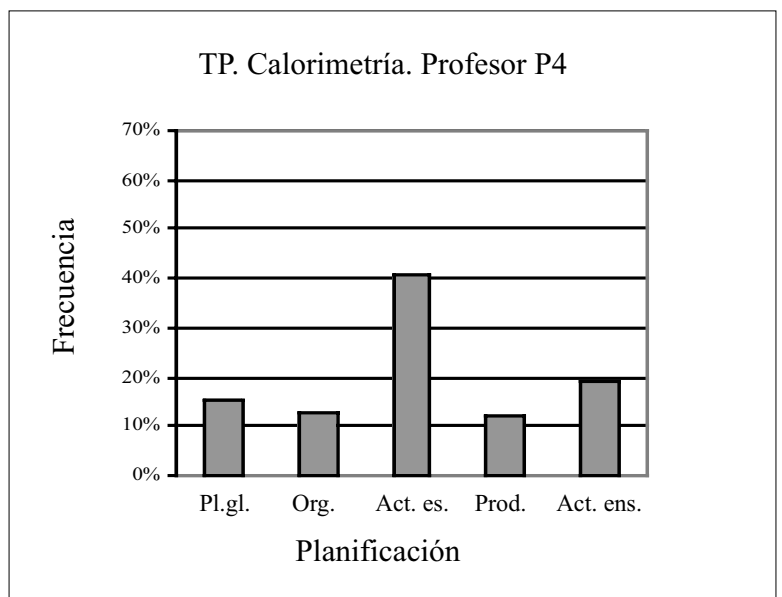

Número total de unidades codificadas: 39

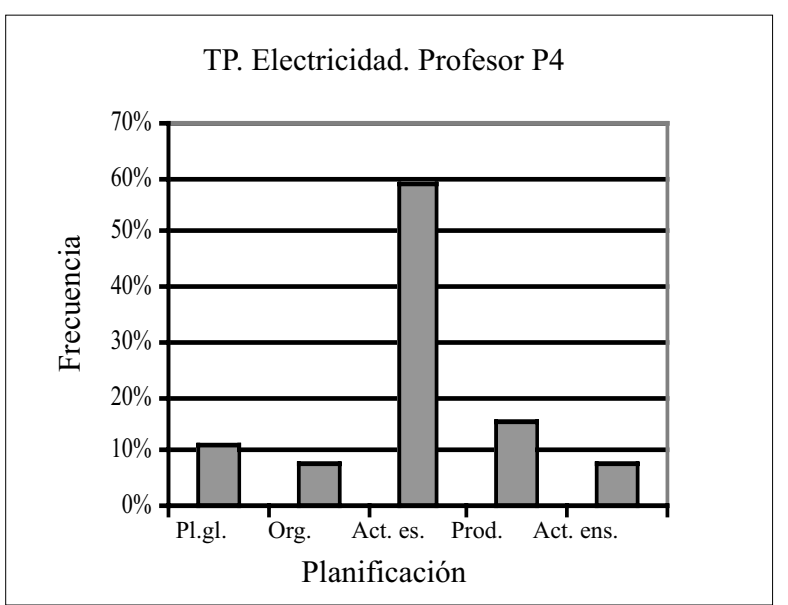

Número total de unidades codificadas: 39 
Figura 4

Diagramas de las distribuciones de los tipos de «razones» invocadas por los profesores.

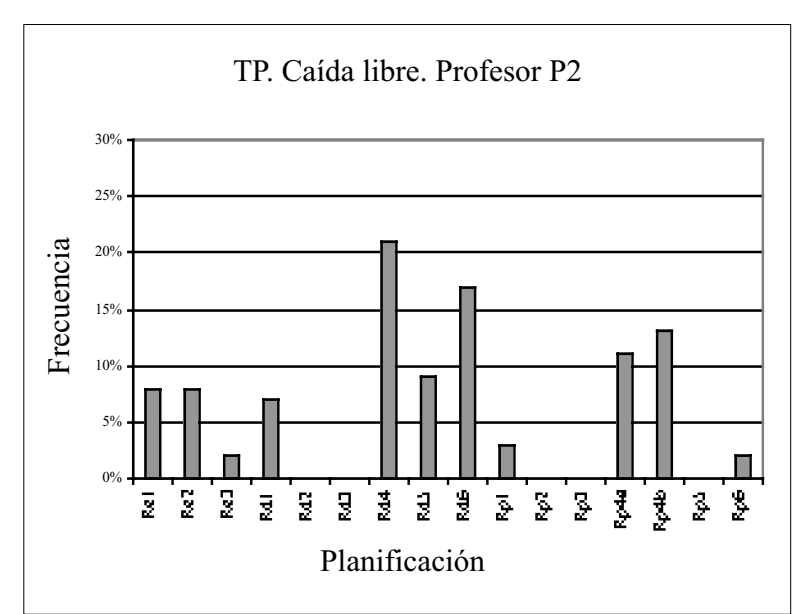

Número total de unidades codificadas: 87

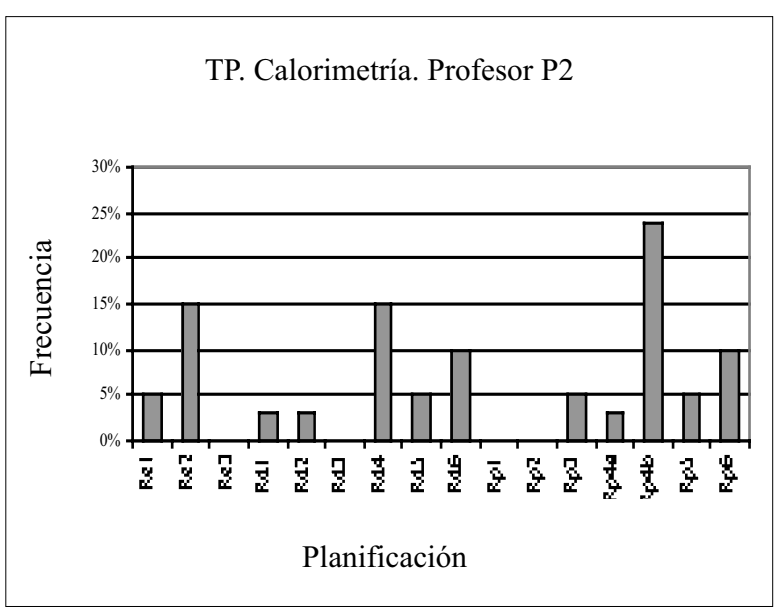

Número total de unidades codificadas: 41

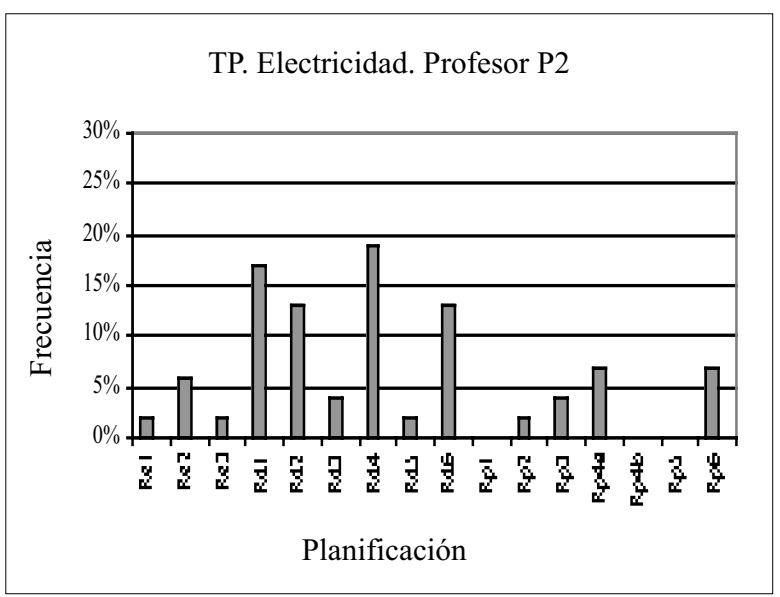

Número total de unidades codificadas: 72

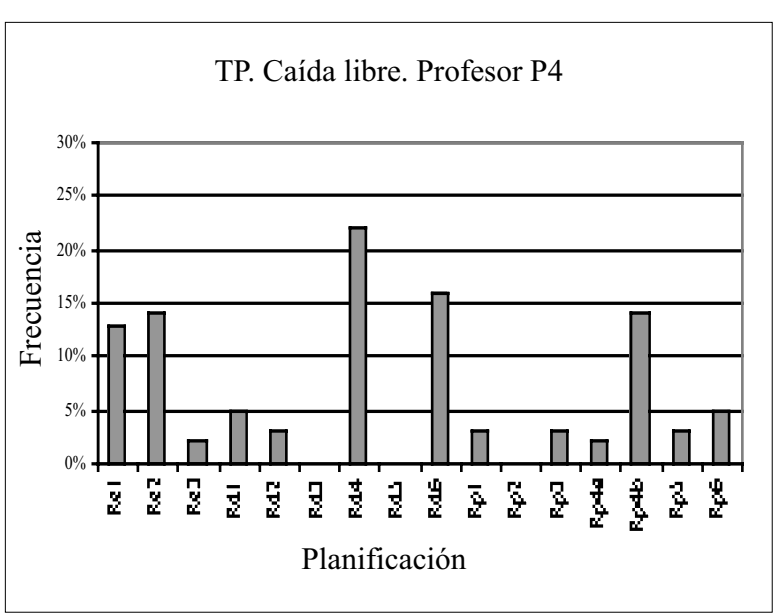

Número total de unidades codificadas: 76

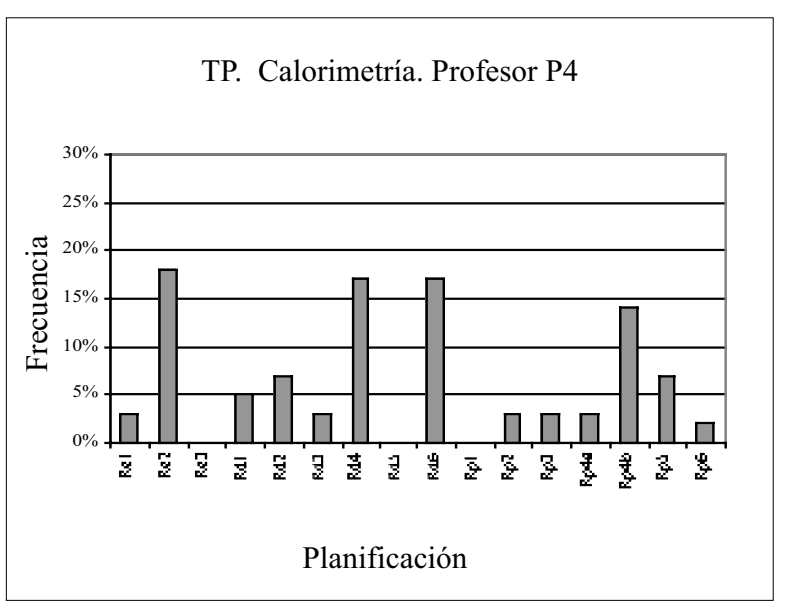

Número total de unidades codificadas: 45

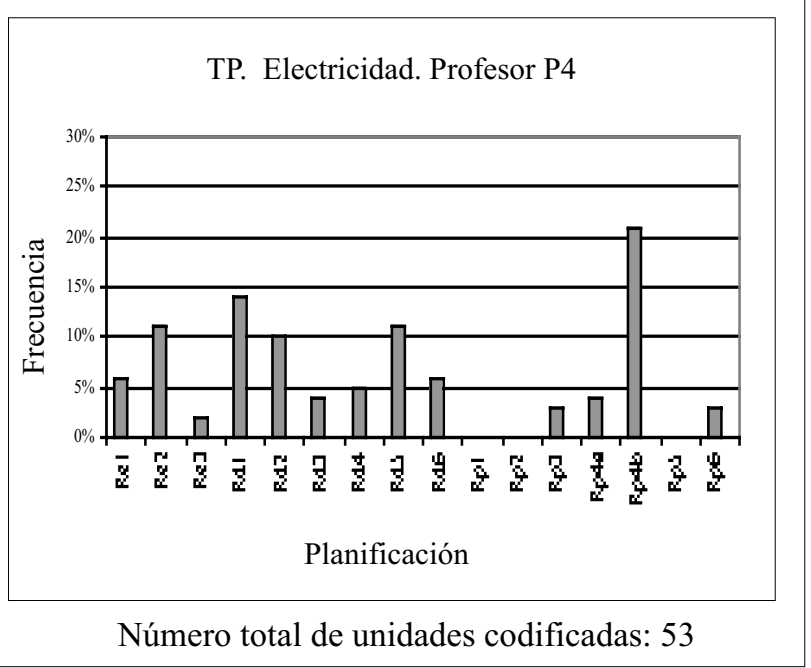


de cada uno de los ítems y recoger las relaciones entre los ítems de planificación y de «razones».

A continuación presentamos un balance de los resultados del análisis en términos de «planificación» primero y después en términos de «razones». Como para cada una de las entrevistas el total acumulado de apariciones de los ítems de «planificación»y de «razones» es inferior a cien y, por lo tanto, su frecuencia es débil, utilizaremos los resultados obtenidos sólo como indicadores de tendencia.

\section{Balance de la planificación}

A fin de hacer más legibles los resultados, los presentamos reagrupando los ítems según los ejes principales de planificación definidos (Fig. 1): planificación global (Pl.gl.), planificación de la organización (Org.), planificación de las actividades de los estudiantes (Act.es.), planificación de la producción (Prod.) y, por último, planificación de las actividades de los profesores (Act.ens.) (Fig. 3).

Vemos así que, si bien para el conjunto de trabajos prácticos todos los aspectos de planificación son cubiertos, la planificación de las actividades de los estudiantes es siempre lo más importante. Este resultado muestra que los profesores tenían efectivamente respuestas para dar y precisiones a desarrollar en lo que apareció como su preocupación principal.

Aparecen diferencias según los temas estudiados, principalmente en el nivel de la organización. Esta primera aproximación no permite determinar qué es, en cuanto a cada tema, lo que induce esas diferencias.

\section{Balance de las razones,justificaciones y argumentaciones}

¿Qué pasa con las razones y las elecciones de los profesores que han llevado a estas planificaciones? Presentamos los resultados para cada sesión de trabajos prácticos (Fig. 4), dando la frecuencia para cada ítem tomado separadamente esta vez, en cada una de las entrevistas previas, a fin de hacer aparecer mejor las razones que se repiten sistemáticamente y aquéllas que, por el contrario, casi no se encuentran.

\section{Las razones de funcionamiento}

En el bloque de «razones de funcionamiento» $(\operatorname{Re} 1, \operatorname{Re} 2 \mathrm{y}$ Re3), notamos que la frecuencia más alta para todos los trabajos prácticos estudiados la tiene el material (Re2). Por otra parte, esta frecuencia siempre está entre las más altas cuando nos referimos al conjunto completo de «razones». Así, por ejemplo, las restricciones ligadas al número de puestos de medición para el estudio de la caída libre explican la importancia que da el profesor P2 a la organización de la sesión:

«Después de hablar de la manipulación, les expliqué que había dos puestos de adquisición. Esto es importante, hace falta que trabajen en grupos de tres [...] esto es lo que pensé hacer con los medios disponibles.»
Las dificultades potenciales en la medición explican igualmente que, para esta misma sesión, el profesor P2 provea una tabla de mediciones a los estudiantes:

«Espero poder sacar algo de esto, pero no es seguro [...] porque he hecho decenas de ensayos y he tenido resultados verdaderamente muy divergentes, muy divergentes.»

Esto está en coherencia con la importancia tomada por el material didáctico, ya señalada, para explicar la persistencia de los hábitos (Hirn, 1995; Couchouron et al., 1996) y el papel de la experiencia en la metodología experimental puesta en marcha en la enseñanza (Joshua y Joshua, 1987) o para caracterizar las experiencias perennes (BécuRobinault y Tiberghien, 1995). Las otras «razones» o bien resultan sensibles a cada situación particular o bien tienen una frecuencia pequeña, casi nula.

\section{Las razones de enseñanza}

En lo que concierne a las «razones» relativas a la enseñanzaaprendizaje de la física ( $\mathrm{Rd} 1, \mathrm{Rd} 2, \mathrm{Rd} 3$ y $\mathrm{Rd} 4)$, una primera comparación de los gráficos muestra la presencia siempre importante (entre un $10 \%$ y un $20 \%$ ) del ítem Rd4, que testimonia que los profesores tienen en cuenta las dificultades de aprendizaje y muestra que ellos conocen y prevén las dificultades de los estudiantes frente al aprendizaje de la física:

- Comprender el principio de adquisición de las mediciones en un montaje nuevo:

«En el momento en que ellos hagan la prueba para verificar si el Magnum funciona, verán las celdas que se obturan [...] pasando el dedo. Se les hace pasar el dedo para que comprendan bien este tema de las celdas emisoras y receptoras.»

- Tener en cuenta las dificultades conceptuales:

«Verdaderamente está en relación con la unidad temática [...] y con la pregunta de antes para tratar de relacionarlo con lo que se ha visto sobre el principio de inercia.»

La comparación de los gráficos pone igualmente en evidencia la casi total ausencia, en las seis entrevistas, del ítem $\mathrm{Rd} 3$, relativo a la enseñanza de los procedimientos de investigación científica: de hecho, ninguna de las seis sesiones de trabajos prácticos tuvo como objetivo enseñar a los estudiantes a realizar un proceso de investigación experimental. Por último, en lo que concierne a la enseñanza de contenidos conceptuales y procedimentales ( $R d 1$ y $R d 2)$, las frecuencias de estos dos ítems aparecen dependientes de la situación.

A propósito de las «razones» en relación con las elecciones personales de los profesores ( $\mathrm{Rd} 5, \mathrm{Rd} 6)$, podemos notar que la aparición del ítem $\mathrm{Rd} 5$ relativo a la construcción de un referente (gráfica, modelo matemático o resultado numérico esperado de cada grupo de estudiantes durante el trabajo práctico) es importante cuando lo que sigue del curso está construido sobre esos elementos: la construc- 
ción de este referente común a los estudiantes y al profesor es entonces un objetivo didáctico preciso como en el ejemplo citado a continuación (caída libre, profesor P2):

«Me parece que los estudiantes saben de qué hablo, puesto que, aunque sea poco, han trabajado... ¿cómo decirte?, se han encontrado con la dificultad experimental, han visto lo que era. Entonces, cuando yo voy a preparar el curso, atención: es una caída modelizada, ideal, etc. Ellos sabrán de qué les hablo porque se encontrarán con dificultades; no es sencillo [...].»

En cuanto a los argumentos correspondientes a las elecciones personales de los profesores de física, todos agrupados en Rd6, están a priori correlacionados con una imagen de la enseñanza de la física. Las elecciones «para la física» mencionadas en las diferentes entrevistas son muy variadas, como por ejemplo:

- Hacer la sesión de trabajos prácticos antes de la clase teórica:

«Sí, me gusta cuando funciona, cuando funciona en la progresión. Me gusta verlo primero en el TP, introducirlo en el TP y explotarlo después, lo prefiero [...] (caída libre, profesor P2).»

- Establecer una relación matemática a partir de mediciones:

«Tenía ganas de mostrar que había una relación, que esto varía pero que no varía por azar, que hay leyes, incluso si estas leyes no se pueden justificar con los conocimientos que se tienen (caída libre, profesor P4).»

- Hacer que los estudiantes usen folletos técnicos para poner en acción un material nuevo:

«Trato también de darles extractos de, bueno, folletos técnicos $[\ldots]$ pienso que es importante que ellos sean capaces de leer cosas un poco técnicas [...] (caída libre, profesor P4).»

Podemos observar que el sello de cada profesor (frecuencias Rd5 + Rd6) está claramente impreso. Esto está en coherencia con el diseño de los seis trabajos prácticos, que son creaciones originales (o adaptaciones) realizadas por ellos.

Sobre el conjunto de los ítems que conciernen a la previsión del desarrollo de la sesión (Rp1, Rp2, Rp3 y Rp4), notamos la presencia masiva de Rp4 en todas las entrevistas. Este ítem agrupa las dificultades que los profesores pueden encontrar durante la sesión (dificultades ligadas al material (Rp4a), a los estudiantes (Rp4b), como su comportamiento y su grado de compromiso con la tarea) y las incertidumbres acerca de su desarrollo:

«Para la medición de la masa del hielo, a toda costa quieren ponerlo sobre la balanza. Bueno, me espero cualquier cosa (calorimetría, profesor P2).»

«No sé para nada qué se imaginarán [...] de hecho, son estudiantes muy dispersos porque no son muy buenos en la parte teórica, pero pueden tener ideas, quizás tendrán ideas (caída libre, profesor P4).»

La frecuencia del ítem Rp4 muestra que, en la proyección que los profesores hacen del desarrollo del trabajo práctico, este elemento está muy presente y debe adaptarse a las dificultades que aparecen (Tochon, 1989). Por último, las «razones» que tienen que ver con los objetivos generales de enseñanza (Rp5) aparecen poco en este balance que hemos realizado: los objetivos disciplinares son los que guían esencialmente la elaboración de los trabajos prácticos de física. Entre los pocos ítems relevados en «otras» razones (Rp6), no hemos encontrado ninguna referencia a la práctica científica.

Los resultados del análisis de las entrevistas en términos de planificación y de «razones» muestran a la vez la importante variedad de parámetros que los profesores deben gestionar y las elecciones que ellos realizan. Proponemos a continuación un modelo para la construcción de una sesión de trabajos prácticos que sintetiza estos resultados.

\section{SÍNTESIS: UN MODELO PARA LA CONS- TRUCCION DEL TRABAJO PRACTICO}

Entre las «razones» que hemos listado y analizado en este trabajo, un conjunto aparece impuesto a los profesores y representa «restricciones institucionales»: se trata de tomar en cuenta el programa, la gestión del material (calidad, número, etc.), las aulas disponibles, los horarios (duración de las sesiones, alternancias). En todas las otras «razones»-tales como «la sesión de trabajos prácticos permitirá construir un referente», «los estudiantes trabajarán de manera autónoma», «se han de tener en cuenta las dificultades»)-, intervienen las elecciones de los profesores y sus propias referencias: conocimiento acerca de la clase, de los estudiantes y de la enseñanza. Así, para la situación de trabajos prácticos que estudiamos a partir del análisis de las entrevistas, la estructura de la construcción del trabajo práctico por parte de los profesores resulta bipolar: la «gestión de las restricciones» constituye uno de los polos y el «modelo para la puesta en marcha de la enseñanza» representa el otro. Este último abarca, para cada profesor, el modelo que él o ella se hace de la enseñanza científica y el modelo de estudiante que ha construido, ya sea de manera más bien general o más específicamente para la clase que tiene a su cargo durante el año. El modelo para la construcción se tornaría entonces según vemos en la figura 5.

La flecha profesor $\rightarrow$ actividades planificadas representa aquí la actividad de construcción de los profesores. Hemos inscrito el conjunto en un recuadro que representa el marco institucional de la clase, ya que, si bien no hay duda de que los programas, horarios y restricciones materiales forman parte del marco institucional de la enseñanza, nos parece que los profesores son también miembros de esta institución y que el modelo de estudiante del que hemos hablado no es el de un estudiante-individuo-aprendiz, sino más bien el de un estudiante en un sentido más genérico, el «estudiante de la clase X». Este esquema permite, pues, analizar los vínculos que los profesores establecen entre 
Figura 5

Modelo de construcción de un trabajo práctico.

Marco institucional de la clase

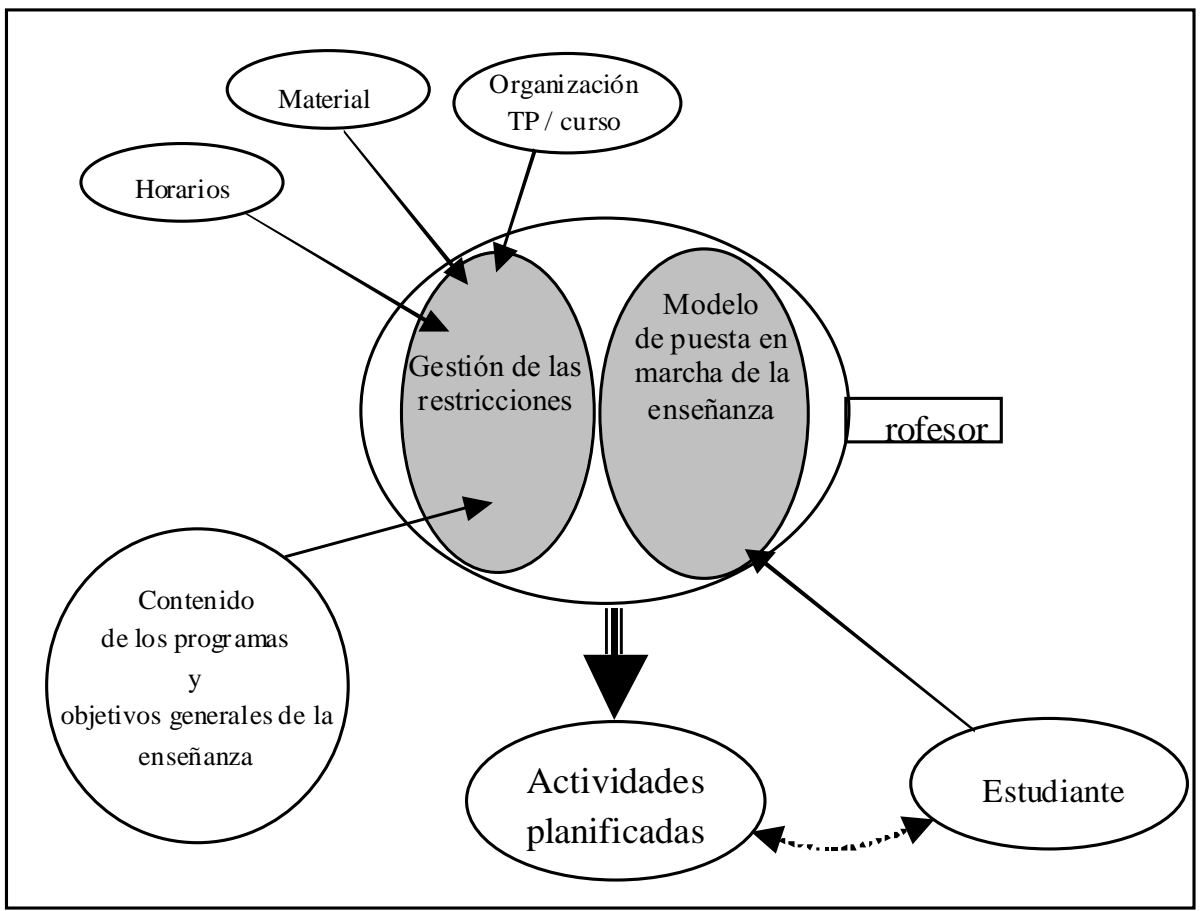

las actividades que planifican y las razones y elecciones que ellos indican para cada sesión de trabajos prácticos.

Este análisis muestra la complejidad del sistema que los profesores gestionan. A la luz de nuestro trabajo, los profesores aparecen como «profesores-constructores», es decir, como profesores ingenieros que, para elaborar las sesiones de trabajos prácticos, deben tener en cuenta parámetros muy diferentes: límites de los programas, planificación de la enseñanza -incluyendo la evaluación de los aprendizajes- y organización específica de la sesión -incluyendo las restricciones particulares a las actividades experimentales (seguridad, fiabilidad del material, etc.).

\section{REFERENCIAS BIBLIOGRÁFICAS}

AYÇAGUER-RICHOUX, H. (2000). «Rôles des expériences quantitatives dans l'enseignement de la physique au lycée». Tesis en didáctica de la física. París 7, Universidad Denis Diderot, $205 \mathrm{pp}$. <http://www.inrp.fr/Tecne/TecneDoc/ Docenligne.htm $>$
El reconocimiento de esta dimensión profesional en la formación inicial es una apuesta central (Porlán y Rivero, 2001) y, además de los trabajos que han contribuido a la reflexión sobre la formación del profesorado -en las dimensiones epistemológica (Robardet, 1998) y didáctica (Saint-Georges, 1998)-, nos parece también esencial que la didáctica de las ciencias se interese por la práctica de los profesores.

\section{NOTA}

Este artículo ha sido traducido del francés por Agustín AdúrizBravo. 
BECU-ROBINAULT, K. y TIBERGHIEN, A. (1995). Un exemple d'articulation entre recherches d'après manuels et programmes et recherches menées dans le cadre d'un enseignement réel, en Mary, G. y Kaminsky, W. (eds.). Actes du cinquième Séminaire de Recherche en Didactique des Sciences Physiques. Université de Reims y IUFM de Reims, 15-17 octubre 1995, pp. 71-85.

BELHOSTE, B., GISPER, H. y HULIN, N. (1996). Les sciences au lycée. Un siècle de réformes des mathématiques et de la physique en France et à l'étranger. París: INRP, Vuibert.

BENEY, M. y SÉRÉ, M.G. (2001). Entre réussir et comprendre ou de l'effet des consignes opératoires sur la compréhension des procédures de mesurage en TP de physique de $1 \mathrm{r}$. cycle universitaire. Didaskalia, 19, pp. 9-37.

BROUSSEAU, G. (1986). Fondements et méthodes de la didactique des mathématiques. Recherches en Didactique des Mathématiques, 7(2), pp. 33-105.

BROUSSEAU, G. (1988). Le contrat didactique: le milieu. Recherches en Didactique des Mathématiques, 9(3), pp. 309-336.

BULLETIN OFFICIEL DE L'ÉDUCATION NATIONALE (1999). Physique-chimie, Classe de seconde, Nouveau Programme applicable à compter de l'année scolaire 20002001, 6, pp. 7-23.

COMITI, C., GRENIER, D. y MARGOLINAS, C. (1995). Niveaux de connaissances en jeu lors d'interactions en situation de classe et modélisation de phénomènes didactiques, en Arsac, G., Gréa, J., Grenier, D. y Tiberghien, A. Différents types de savoirs et leur articulation. Grenoble: La pensée sauvage, pp. 93-127.

COUCHOURON, M., VIENNOT, L. y COURDILLE, J.M. (1996). Les habitudes des enseignants et les intentions didactiques des nouveaux programmes d'électricité en classe de quatrième. Didaskalia, 8, pp. 81-96.

GUILLON, A. (1995). Démarches scientifiques en travaux pratiques de physique de DEUG à l'université de CergyPontoise. Didaskalia, 7, pp. 113-127.

HASLAM, F. y GUNSTONE, R. (1998). The influence of teachers on student observation in science classes, en Annual Meeting of the National Association for research in Science teaching. San Diego.

HEWSON, P. y OLSEN, T. (1994). Qualitative physics and student's ideas: a physics teacher's story. International Journal of Science Education, 16(5), pp. 563-573.

HIRN, C. (1995). Comment les enseignants de sciences physiques lisent-ils les intentions didactiques des nouveaux programmes d'optique de la classe de quatrième. Didaskalia, 6, pp. 39-54.

INSPECTION GÉNÉRALE DE L'ÉDUCATION NATIONALE (1996). Groupe de Physique et Chimie. La place de l'expérimental dans l'enseignement de la physique et de la chimie.

JOHSUA, M.A. y JOHSUA, S. (1987). Les fonctions didactiques de l'expérimental dans l'enseignement scientifique (première partie). Recherche en Didactique des Mathématiques, 8(3), pp. 231-266.
LEACH, J. y PAULSEN, A. (1999). Practical work in science education. Recent Research Studies. Roskilde University Press.

LUC, C. y DUREY, A. (1997). Modèle et modélisation dans les séquences de travaux pratiques sur le haut-parleur en classe de seconde. Didaskalia, 11, pp. 39-72.

NOTT, M. y SMITH, R. (1995). «Talking your way out of it », «rigging » and «conjuring»: what science teachers do when practicals go wrong. International Journal of Science Education, 17(3), pp. 399-410.

OLSEN, T., HEWSON, P. y LYONS, L. (1996). Preordained science and student autonomy: the nature of laboratory tasks in physics classrooms. International Journal of Science Education, 18(7), pp. 775-790.

PERRIN-GLORIAN, M.J. (1994). Théorie des situations didactiques: naissance, développement, perspectives, en Artigue, M., Gras, R., Laborde, C., Tavignot, P. Vingt ans de didactique des maths en France. Grenoble: La pensée sauvage, pp. 97-147.

PORLÁN ARIZA, R., RIVERO GARCÍA, A. y MARTÍN DEL POZO, R. (2000). El conocimiento del profesorado sobre la ciencia, su enseñanza y aprendizaje, en Perales Palacio, F.J. y Cañal de León, P. (eds.). Didáctica de las ciencias experimentales, pp. 507-533. Alcoy: Marfil.

PORLÁN ARIZA, R. y RIVERO GARCÍA, A. (2001). Nature et organisation du savoir professionnel «souhaitable». Aster, 32, pp. $221-251$.

RICHOUX, H. (1998). Quels sont les rôles des expériences quantitatives dans l'enseignement de la physique au lycée? en Actes du $\sigma^{e}$ séminaire national de recherche en didactique de la physique, de la chimie et de la technologie. Lyon: LIRDHST, pp. 243-249.

ROBARDET, G. (1998). La didactique dans la formation des professeurs de sciences physiques face aux représentations sur l'enseignement scientifique. Aster, 26, pp. 31-58.

ROLETTO, E. (1998). La science et les connaissances scientifiques: point de vue de futurs enseignants. Aster, 26, pp. 11-30.

SAINT-GEORGES, M. (1998). Formation des professeurs de sciences physiques par la didactique. Didaskalia, 13, pp. 57-80.

SÉRÉ, M.G. (1998). Improving science education: issues and research on innovative empirical and computer-based approaches to labwork, en Europe, final report, European Commission, DG XII, TSER programme. Bruselas.

TOCHON, F. (1989). À quoi pensent les enseignants quand ils planifient leurs cours? Revue Française de Pédagogie, 86, pp. 23-33.

TSOUMPELIS, L. y GRÉA, J. (1995). Essai d'application de la théorie des situations en sciences physiques-apprentissage de la concentration molaire en classe de première $\mathrm{S}$ (grade 11 US). Recherche en Didactique des Mathématiques, 15(2), pp. 63-108.

VAN DRIEL, J.H. y VERLOOP, N. (1999). Teachers' knowledge of models and modelling in science. International Journal of Science Education, 21(11), pp. 1141-1153.

[Artículo recibido en junio de 2001 y aceptado en septiembre de 2002.] 